
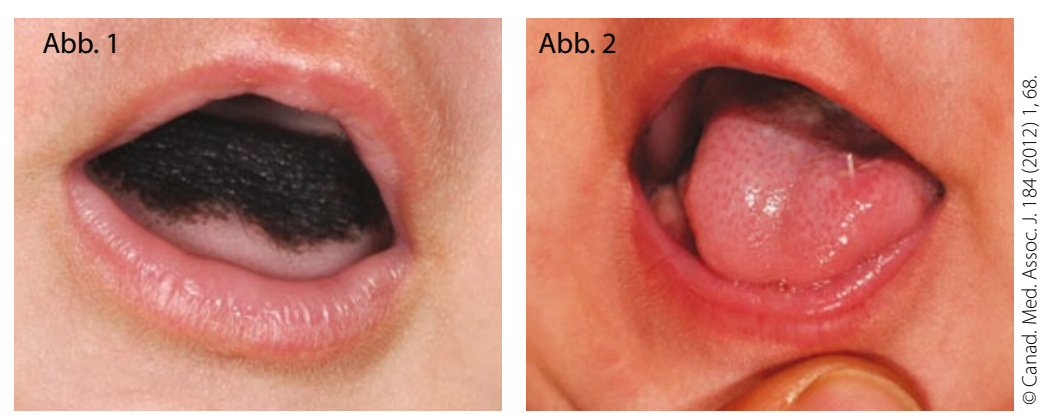

\section{Ein acht Wochen alter Säugling wurde in der dermatologischen Ambulanz wegen einer seit vier Wochen von den Eltern beobach- teten Schwarzverfärbung der Zunge vorgestellt (Abb. 1).}

- Die Anamnese des Kindes war unauffällig. Die Familienanamnese erbrachte keinen Anhalt für ein Peutz-JeghersSyndrom, eine Neurofibromatose von Recklinghausen oder einen Morbus Addison.

Zum Ausschluss einer Melanosis wurde eine Biopsie der Zunge vorgenommen. Drei Wochen später hatte sich die Schwarzverfärbung bei dem Kind ohne jede Therapie vollständig zurückgebildet (Abb. 2). In der Biopsie fanden sich eine gemischte Flora aus Bakterien und Pilzen, aber keine Melanozyten.

Abb. 1 Schwarzverfärbung der Zunge eines acht Wochen alten Säuglings. Abb. 2 Völliges Verschwinden der Schwarzverfärbung drei Wochen später.

Die Lingua villosa nigra, ist eine schmerzlose, gutartige Zungenveränderung mit einer ungewöhnlichen, braunschwarzen Verfärbung der Zungenoberfläche. In der Regel tritt die Schwarzfärbung bei über 40-jährigen Personen mit schlechter Oralhygiene, starkem Nikotinabusus und Anwendung von Antibiotika auf. Bei Kindern gehört die schwarze Haarzunge dagegen zu den absoluten Raritäten.

\section{- A. Körber und N. Voshege}

(Korres.: Dr. Andreas Körber, antikoerper@ hotmail.com) Black hairy tongue in an infant. Canad. Med. Assoc. J. 184 (2012) 1, 68.

\section{Kommentar}

Zur Behandlung der schwarzen Haarzunge werden reichliches Trinken und eine Anregung der Speichelsekretion empfohlen. Auch das Einstellen des Rauchens und das Bürsten der Zunge mit einer weichen Zahnbürste nach Applikation von $40 \%$ Urea, die topische Anwendung von Retinoiden oder Salizylsäure sollen die Rückbildung des Belags beschleunigen. Bei Kindern ist die schwarze Haarzunge meistens selbst limitiert und verschwindet innerhalb einiger Wochen. Es dürfte aber nicht leicht sein, die Eltern über diese Zeit hinweg ohne besondere Therapiemaßnahmen zu vertrösten.

H. S. FÜESSL =

\title{
Zwischen Erfahrung und Zittrigkeit
}

\section{Das Minimum einschlägiger Kompli- kationen nach Thyreoidektomie liegt bei etwa 20 Jahren chirurgischer Tätigkeit, danach überwiegen wahr- scheinlich die Defizite des körper- lichen Abbaus die Erfahrung.}

- Am Beispiel zweier einschlägiger Komplikationen der Schilddrüsenchirurgie zeigte eine multizentrische prospektive Querschnittsuntersuchung an fünf akademischen Lehrkrankenhäusern in Frankreich, dass es offensichtlich einen Zeitpunkt gibt, an dem sich die Lebenslinien zwischen Erfahrung und physischer Leistungsfähigkeit kreuzen. Im Lauf eines Jahres führten hier 28 Chirurgen 3574 totale Thyreoidektomien durch. Insgesamt betrug die Rate von Recurrensparesen 2,08\% und die eines postoperativen Hypoparathyreoidismus $2,69 \%$. In einer multivariaten Analyse ergab sich, dass eine operative
Tätigkeit von 20 Jahren und länger mit einem deutlich erhöhten Risiko für beide Komplikationen einherging. Die Odds Ratio für die Recurrensparese betrug dann 3,06, für den Hypoparathyreoidismus sogar 7,56.

Die Performance der Chirurgen wies eine konkave Assoziation mit der Länge der Erfahrung und dem Alter auf. Chi- rurgen im Alter zwischen 35 und 50 Jahren hatten signifikant niedrigere Komplikationsraten als ihre jüngeren und älteren Kollegen.

\section{- A. Duclos et al.}

Influence of experience on performance of individual surgeons in thyroid surgery: prospective cross sectional multicentre study. Brit. Med. J. 344 (2012) d8041

\section{Kommentar}

Den Autoren der Untersuchung gebührt Respekt, da es sich um ein heißes Eisen handelt, das in der nach wie vor hierarchisch gegliederten Medizin nicht gerne diskutiert wird. Die Ursache für die erhöhte Komplikationsrate bei sehr jungen Chirurgen liegt wohl auf der Hand. Warum allerdings über 50-jährige Chirurgen so viel schlechter abschneiden als ihre mittelalten Kollegen muss auch nach dieser Studie Gegenstand der Spekulation bleiben. Neben einem gewissen physischen und kognitiven Abbau kämen ja noch andere Gründe infrage, zum Beispiel die erhöhte Belastung durch administrative Aufgaben, die vor allem auf den Schultern älterer Kollegen liegt, eine möglicherweise nicht mehr aktuelle handwerkliche Ausbildung oder auch eine gewisse Hybris mit Überschätzung der eigenen Fähigkeiten. Jeder in dieser Situation prüfe sich selbstkritisch!

H. S. FÜESSL = 